\title{
Your Microbes at Work: Fiber Fermenters Keep Us Healthy
}

The gut houses trillions of microbes. They eat what you eat. Many specialize in fermenting the soluble fiber in legumes, grains, fruits and vegetables. Certain microbial species are adept at colonizing the mucous layer of the gut. Mucus contains antimicrobial substances that keep the microbiota at a slight distance. But it also contains sugars such as those found in breast milk. Some microbes, often the same ones that specialize in fermenting fiber, can use these sugars as sustenance when other food is not available. The by-products of fiber fermentation nourish cells lining the colon. Some by-products pass into the circulation and may calibrate our immune system in a way that prevents inflammatory disorders such as asthma and Crohn's disease.

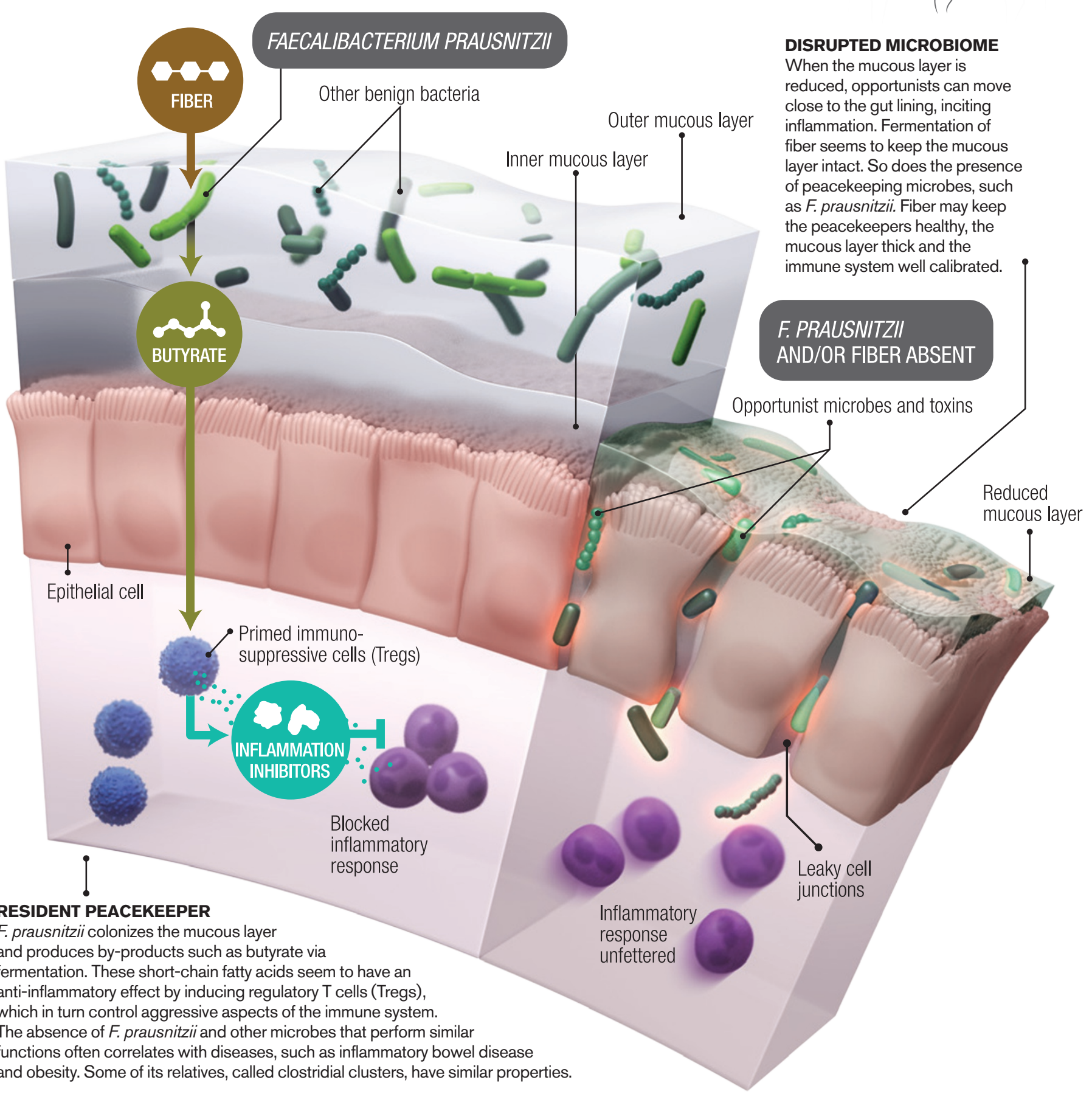

